\title{
Análisis contrastivo de las traducciones de Mafalda en francés por Jacques y Anne-Marie Meunier
}

\author{
Contrastive analysis of Mafalda's translations in \\ French by Jacques and Anne-Marie Meunier
}

\author{
Carolina Munguía González \\ Escuela de Educación y Desarrollo Humano \\ Universidad De La Salle Bajío, México
}

\section{Resumen}

Las historietas de Mafalda han traspasado las barreras del idioma y de la cultura hasta en las regiones menos esperadas del mundo, tal ha sido la influencia de este personaje que es elegido para acompañar la campaña de la Declaración de Los Derechos del Niño para la Unicef un par de años después de ser publicada. A pesar de su popularidad, existen escasos trabajos de investigación y análisis sobre sus distintas traducciones. Este artículo versa sobre el análisis contrastivo realizado del primer tomo de Mafalda (1966) y su homólogo en francés traducido por Jacques y Anne-Marie Meunier, Mafalda et ses amis (1984) tras casi dos décadas; enfatizando, principalmente, en los elementos culturales de ambos idiomas e identificando las dificultades de traducción de los aspectos socioculturales y lingüísticos, utilizando una metodología cualitativa de interpretación. Se constata que, aun cuando el trabajo de los Meunier amerita reconocimiento por su habilidad de transferir algunos elementos culturales y humorísticos en nuevos contenidos auténticos significativos para el público meta, las traducciones pierden, en cierta medida, algunos de los elementos que caracterizan la obra.

Palabras clave: análisis contrastivo, traducción cultural, tiras cómicas, transcreación

\begin{abstract}
Mafalda's comic strips have transcended linguistic and cultural barriers even in the least expected regions from all over the world, such was its influence that a couple of years after it was published this character was chosen to host the Declarations of the Rights of the Child campaign for Unicef. Despite the popularity of the comic, research work and analysis about its several translations is scant. This article addresses a contrastive
\end{abstract}


analysis done to the first volume of Mafalda (1966) and its French counterpart translated by Jacques and Anne-Marie Meunier, Mafalda et ses amis (1984) two decades later; emphasizing, particularly, on the cultural elements of both languages and identifying the difficulties of the translation, sociocultural and linguistic, using a qualitative methodology of data interpretation; evidencing that even though the Meunier's translations merit recognition due to their ability to transfer some of the cultural and humorous elements into new authentic content meaningful to the target audience, these translations loose to some extent some of the elements that characterizes the comic.

Key words: contrastive analysis, cultural translation, comic strips, transcreation

\section{Introducción}

L a definición de traducción ha cambiado drásticamente a través de la historia, especialmente en las últimas décadas. El proceso de traducir nace probablemente de la intuición misma del ser humano para buscar diferentes maneras de transmitir la misma idea en diferentes contextos. En sus inicios, el principal enfoque de la traducción era el de transferir un texto a otro idioma sin ninguna alteración, es decir, una traducción literal. Este enfoque parecía ser bastante coherente para su época; no obstante, hoy en día, es de conocimiento general que, en la práctica, esta labor es mucho más compleja.

Dentro de este campo, existen diversos paradigmas de lo que significa transmitir un mensaje de una lengua o de una cultura a otra; sin embargo, podría afirmarse que finalmente "la traducción es siempre reflejo del tipo de sociedad que la genera" (Claramonte, 2009, p. 49), es decir, es el resultado del entendimiento de un mundo y conlleva, invariablemente, una relación intrínseca con los constructos que emanan de ella. Es posible anticipar algunos de los retos de una traducción si se identifican previamente los paradigmas culturales y lingüísticos de ambas, la lengua origen y meta.

\section{La traducción cultural}

Las teorías occidentales más importantes, que surgen a partir de la segunda mitad del siglo XX, pueden enlistarse en seis conceptos centrales: "la equivalencia, la finalidad, la descripción, el indeterminismo, la localización y la traducción cultural" (Pym, 2016, p. 7). Este trabajo hace especial énfasis en la traducción cultural debido a las características particulares de una historieta tan cargada de elementos culturales como lo es en este caso Mafalda. No obstante, es también precisa la ayuda de diversas teorías subyacentes como la de equivalencia o la de finalidad, por mencionar algunas. La traducción cultural responde a las necesidades de un mundo globalizado, producto de procesos culturales sumamente complejos.

\section{La traducción de las tiras cómicas.} La traducción de historietas es un campo relativamente moderno. Nace de la necesidad misma de transferir otro tipo de contenido (diferente al del ámbito literario o del jurídico) a otra lengua pero, aún más, a otro contexto cultural. La misma naturaleza de las tiras cómicas permite la flexibilidad de la traducción e incita, además, a la creatividad del traductor, el ingenio 
y el humor dentro de los sistemas del lenguaje, como el juego de palabras y la transcreación, por mencionar algunos retos (Buzelin, 2004).

En el caso específico de Mafalda, su autor Joaquín Salvador Lavado Tejón alias Quino nos muestra a una niña de seis años de clase media en Argentina de los años sesenta. Más adelante, esta pequeña niña se transforma en un personaje emblemático en todo el mundo, tanto así que en 1977 fue elegida para ser parte de la campaña de la declaración de los de Derechos del Niño para Unicef (Dell'Isola, 2014, p. 14).

Quino cuenta con más de doce volúmenes tan solo de Mafalda en español. Esta pequeña niña se hizo popular en toda América Latina y en otros países tanto de Europa como de Asia. Años más tarde, esta obra traspasó fronteras lingüísticas y culturales para posicionarse como el estandarte de toda una generación (AltaLingua, 2018). Algunas de sus traducciones más emblemáticas han sido al francés, chino, turco, finlandés, portugués e italiano. Aunque no se tienen datos precisos sobre todas y cada una de las traducciones de Mafalda se puede afirmar que estas historietas han sido traducidas en más de treinta lenguajes (Libedinsky, 2001), incluyendo el braille. Sin embargo, poco se habla sobre los trabajos de traducción.

En este artículo, se analizan cuáles son las características de las traducciones de Jacques y Anne-Marie Meunier en francés del volumen 1 de Mafalda con énfasis en la transculturación. Esta pregunta se responde a través de la identificación del léxico, de las estrategias traductológicas y de los elementos culturales en el texto, y al establecer cómo cada autor procede ante estos retos dentro de la traducción.

\section{Metodología}

Partiendo de la noción del concepto lenguaje como sistema complejo de signos y de la idea de constructos culturales y sociales que interactúan para sostener un mensaje, es claro identificar que la investigación presente sigue un enfoque cualitativo.

De esta manera, se comprende y se profundiza en el análisis descriptivo de los elementos lingüísticos y de las culturas en su conjunto insertados en un sistema aún más amplio que comprende a las sociedades involucradas, la francófona y la hispanohablante con sus respectivas características socioculturales, a partir de un análisis comparativo de las tiras cómicas.

Este análisis propuesto conlleva una evaluación del contenido cultural del primer volumen de historietas de Mafalda. Algunos de estos elementos culturales ya han sido tratados en trabajos anteriores, como el de Muñoz y Buesa (2010) donde se comparan las dificultades lingüísticas más comunes en las tiras cómicas de Astérix y sus traducciones al inglés.

Para los fines de este estudio, se ha adaptado un método contrastivo que incluye cuadros comparativos con la información cultural de cada texto para analizar los fragmentos de las tiras. Se hace especial énfasis en los aspectos socioculturales y se amplía en su descripción. Se realiza un conteo de las dificultades de traducción tanto lingüísticas como socioculturales y se señalan sus similitudes y diferencias. En su interpretación se advierten los aciertos y desaciertos de traducción, además de su implicación en términos de transculturación. 


\section{Presentación de resultados}

Se analizaron 240 viñetas tanto del texto original como del texto meta, de las cuales aproximadamente 160 frases y expresiones fueron seleccionadas como portadoras de contenido cultural relevante para el análisis. Los resultados se presentan como proporciones para cada una de las características que se evaluarán, es decir, las dificultades de traducción de aspectos socioculturales y lingüísticos, respectivamente. A continuación, se muestra la comparación de proporción entre autores por nivel de dificultades.

Nivel de dificultades de traducción de aspectos socioculturales. Dentro de esta categoría se desglosan las canciones, costumbres, referencias literarias, políticas e históricas, los dichos o proverbios y las expresiones idiomáticas. Enseguida se muestran los resultados más relevantes para el objetivo de este artículo.

Canciones. Primeramente, se identifican dos canciones dentro del texto. En la primera, se canta "Arrorró mi neeene, arrorró mi sooool..." en el texto origen, y Fais dodo, Colas mon p'tit frère, fais dodo, en el texto meta. Ambas melodías utilizadas culturalmente para cantar a los niños antes de dormir. En la segunda (Figura 1), se adapta la canción "Lamento boricano" de Javier Solís por Que c'est triste Venise de Charles Aznavour, en estas melodías se identifica el sema de la tristeza.
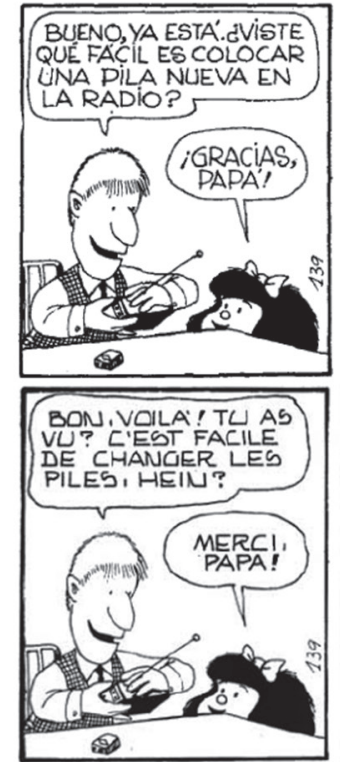
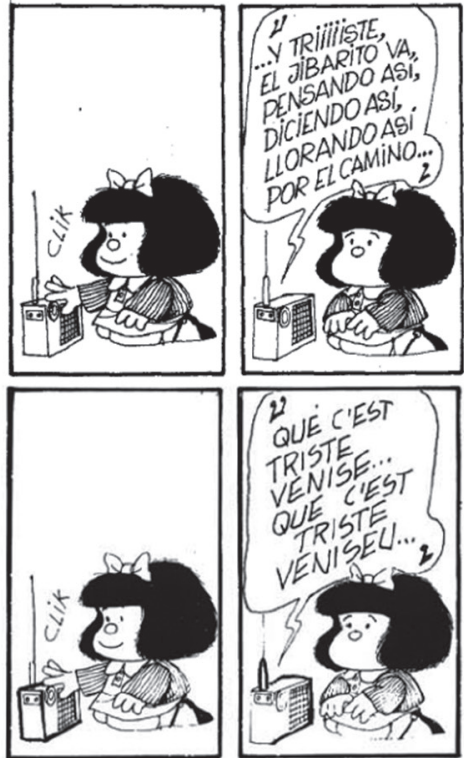

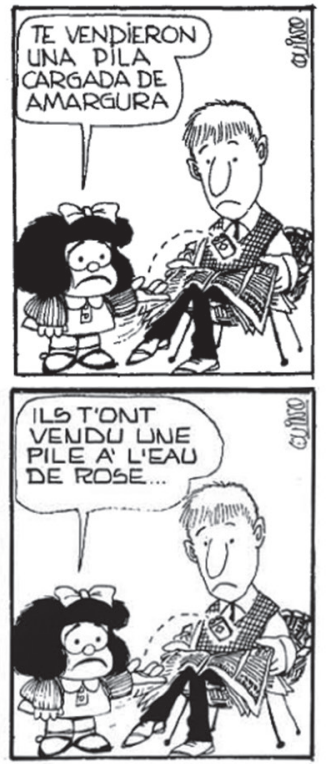

Figura 1. Ejemplo de canciones. Fuente: Mafalda 1 por Joaquín Salvador Lavado y Mafalda intégrale, traducción por Jacques y Anne-Marie Meunier, viñeta número 139. 
Costumbres. En este segmento, se encuentran los elementos relacionados a las costumbres mismas de cada público. La primera viñeta del tomo muestra a Mafalda angustiada preguntando a su madre si el jardín de niños es una carrera. En su traducción, se lee recevoir un diplôme, recibir un diploma, adaptando ligeramente para respetar el sentido de la escolaridad en ambos países.

Por otro lado, la expresión Ceux qui ne mangent pas leur soupe ne grandissent pas! es traducida literalmente de "Los que no toman la sopa no crecen nunca" en español, expresión frecuentemente utilizada por las mamás a sus hijos cuando estos no quieren comer.

Lo mismo sucede más adelante, donde sopa de pescado se traduce como soupe de cresson, platillos representativos de la clase media por su bajo costo. También se identifica una sustitución cultural de la palabra alfajor, dulce típico de Argentina, por meringue, ambos elementos semejantes en apariencia y sentido.

Finalmente, en la viñeta 103 (Figura 2), el papá de Mafalda menciona a su familia "Saqué entradas para ir los tres al teatro infantil", en su traducción al francés se sustituye el concepto teatro infantil por le cirque. Esta referencia sigue más adelante en la misma viñeta cuando menciona "actores" en el texto original y des clowns, des magiciens, des ventriloques en el texto meta haciendo alusión cada uno a su respectivo espacio de entretenimiento.
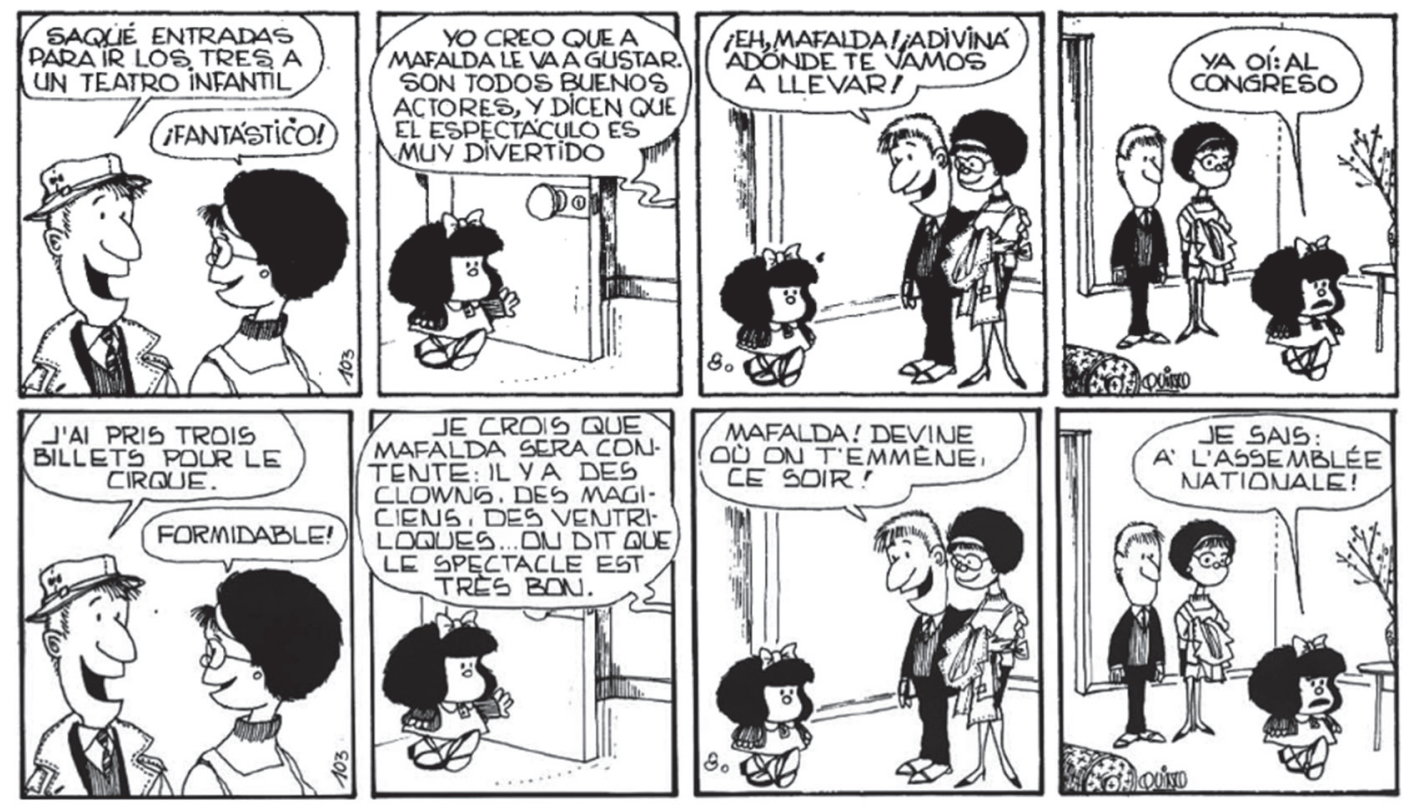

Figura 2. Ejemplo de costumbres. Fuente: Mafalda 1 por Joaquín Salvador Lavado y Mafalda intégrale, traducción por Jacques y Anne-Marie Meunier, viñeta número 103.

Referencias literarias. Tan solo en este volumen se identificaron y analizaron 4 referencias literarias. En la siguiente tabla se muestran los resultados: 
Tabla 1

Muestra contrastiva de referencias literarias

\begin{tabular}{|c|c|c|}
\hline Joaquín Salvador & $\begin{array}{l}\text { Jacques y Anne- } \\
\text { Marie Meunier }\end{array}$ & Comentarios \\
\hline $\begin{array}{l}\text {...si no sabe cómo se } \\
\text { llama "El muchacho". }\end{array}$ & $\begin{array}{l}\text {...s'ils ne savent pas } \\
\text { qui est le justicier. }\end{array}$ & $\begin{array}{l}\text { Se sustituye "El muchacho" por "el justiciero", } \\
\text { concepto descriptivo. }\end{array}$ \\
\hline ¿es James Bond? & Walt Disney !! & $\begin{array}{l}\text { James Bond es uno de los personajes de } \\
\text { ficción más populares del mundo. Walt } \\
\text { Disney, considerado un icono gracias a sus } \\
\text { importantes contribuciones a la industria } \\
\text { del entretenimiento. }\end{array}$ \\
\hline El llanero solitario [...] & $\begin{array}{l}\text { Le cow-boy solitaire } \\
{[\ldots]}\end{array}$ & $\begin{array}{l}\text { Es un personaje ficticio creado por el escritor } \\
\text { Fran Striker. Es un ranger de Texas enmas } \\
\text { carado del viejo oeste de los Estados Unidos } \\
\text { que galopa para enmendar injusticias. }\end{array}$ \\
\hline $\begin{array}{l}\text { Este es Joe Crane un } \\
\text { malvado que les vende } \\
\text { armas a los apaches. }\end{array}$ & $\begin{array}{l}\text { Ça c'est Joe Crane, un } \\
\text { aventurier qui vend des } \\
\text { armes aux apaches. }\end{array}$ & $\begin{array}{l}\text { Aventurier: persona sin escrúpulos, re- } \\
\text { curre a la intriga y al abuso de confianza } \\
\text { para llegar al poder o a conseguir un bien } \\
\text { individual. Timador. }\end{array}$ \\
\hline
\end{tabular}

Fuente: elaboración propia con extractos tomados de la historieta Mafalda 1 de Joaquín Lavado y la traducción al francés de Jacques y Anne-Marie Meunier.

En esta categoría, se identifican algunos retos de traducción que forman parte del repertorio cultural que el traductor debe conocer para adentrarse en su labor. Por ejemplo, en el primer recuadro de la Tabla 1 , se muestra un extracto del texto original donde se hace mención a "El Muchacho", refiriéndose al héroe de una historia. En su traducción, se recurre a un concepto descriptivo de la idea: le justicier, "el justiciero". En el segundo recuadro, se sustituye el personaje James Bond por Walt Disney. Ambos representan a la cultura pop y el surgimiento de la industria del entretenimiento, considerándose inclusive como íconos mundiales.

Referencias políticas. En esta categoría, se analizan las referencias políticas encontradas en el volumen 1 de Mafalda. Se lee "pedazo de extremista" en una de las viñetas, en su traducción el sentido cambia ligeramente a graine d'anarchiste, una adaptación no del todo precisa. Aunque ambas hacen alusión a un tipo de ideología política, estos dos conceptos no son sinónimos en el sentido estricto de sus definiciones. El extremista se define, en política, 
como la persona que tiende a adoptar ideas extremas. En cambio, el anarquista tiene una concepción política fundada sobre el rechazo de toda autoridad. Entonces, resulta que la traducción sí manifiesta una sutil diferencia semántica con el texto original. La viñeta 20 (Figura 3) se identifica también como un ligero cambio en el significado al traducir "sector de la democracia" por parti politique.
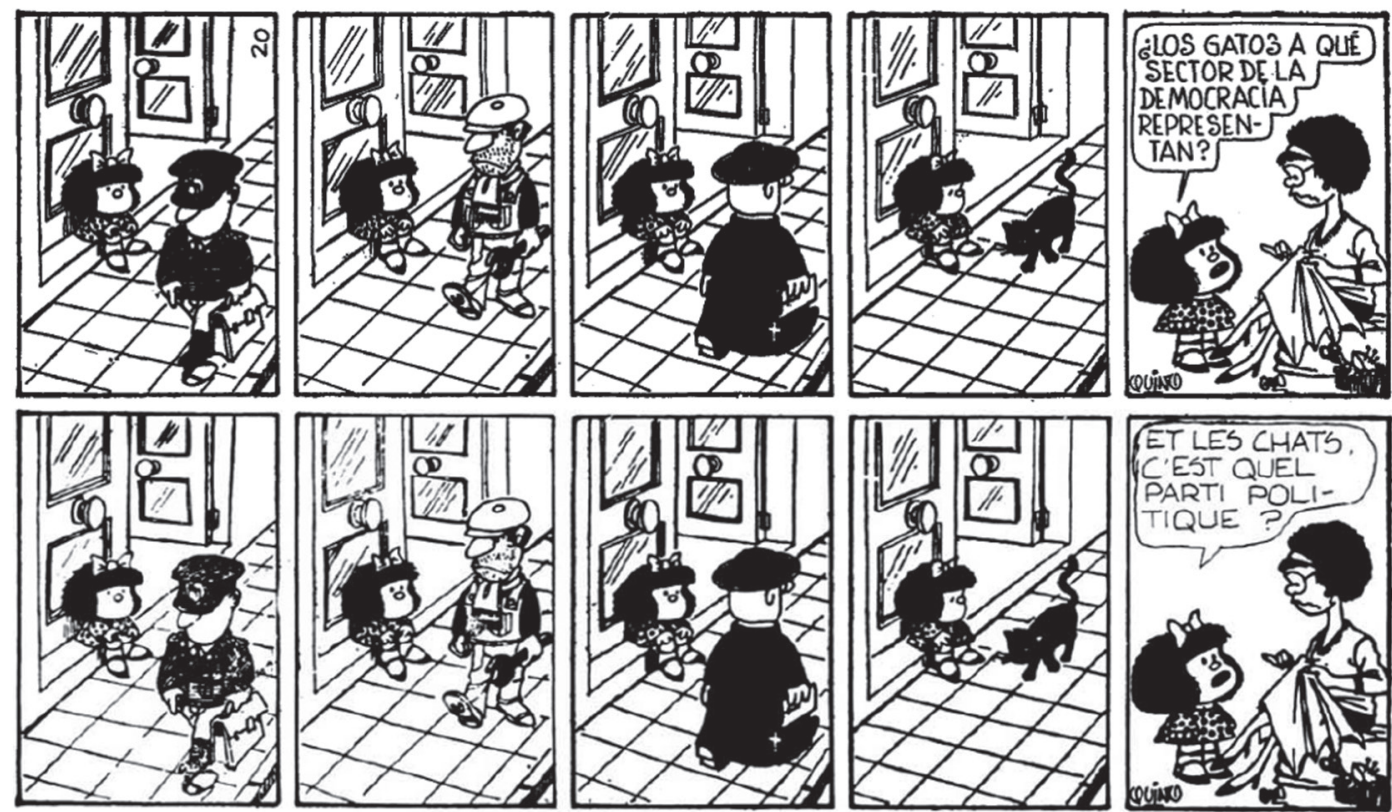

Figura 3. Ejemplo de referencias políticas. Fuente: Mafalda 1 por Joaquín Salvador Lavado y Mafalda intégrale, traducción por Jacques y Anne-Marie Meunier, viñeta número 20.

De regreso en la viñeta 103 (Figura 2), se cierra con "Ya oí: al congreso". Y en francés «Je sais: l'assemblée nationale», la estrategia utilizada es un calco. Ambos conceptos son equivalentes en traducción. Ahora bien, en la expresión “¡Huelga!” encontrada y su traducción Des sous !, esta última está relacionada directamente con la historia política de Francia; se trata de una forma de demanda utilizada principalmente por las personas que se manifestaban debido a las deplorables condiciones económicas. En esta época, las personas exigían mejores condiciones, o sous, que en francés se refiere a una antigua moneda francesa de 5 céntimos.

Referencias históricas. A continuación, los resultados obtenidos de las referencias históricas identificadas en ambos textos. La segunda viñeta del tomo nos muestra a Mafalda diciendo "¡Con todo el campo que tienen aquí las vacas!... ¿Por qué demonios también ellas se van al extranjero?”. Los traductores se deciden por una traducción prácticamente literal: Avec toute la place qu'elles ont ici les vaches... 
Pourquoi diable, elles aussi, elles partent à l'étrangère? Se trata de una clara alusión a La Noche de los Bastones Largos en Argentina (1966), evento caracterizado por representar la fuga de cerebros de la época. La viñeta 122
(Figura 4) es un caso interesante a señalar con múltiples expresiones traducidas cargadas de sentido histórico, las cuales son mayormente resueltas con adaptaciones en la cultura meta.
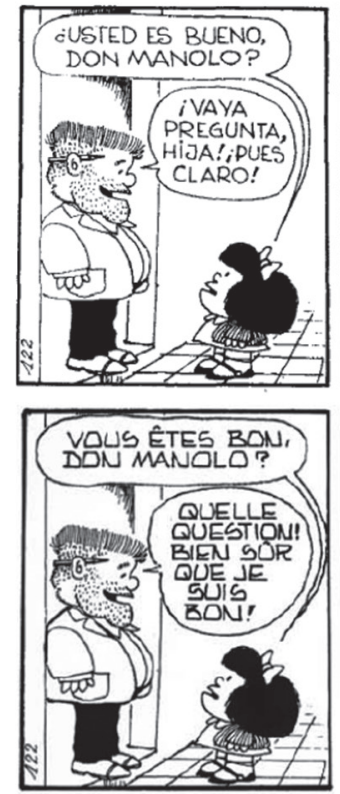
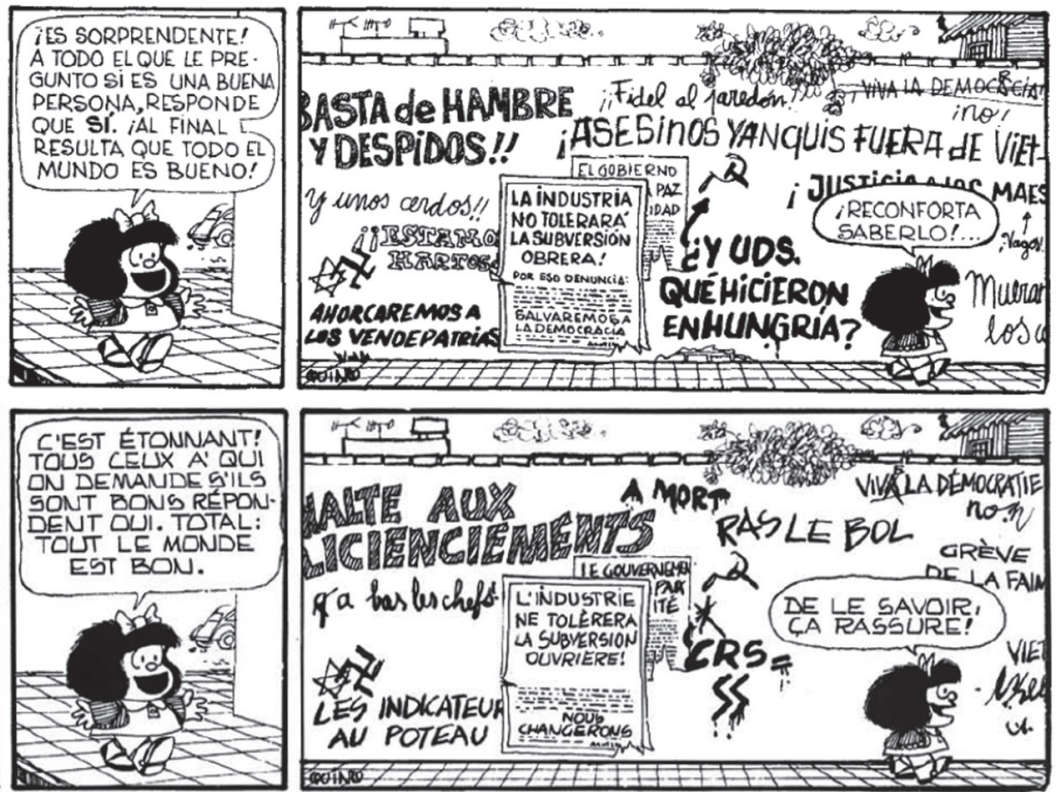

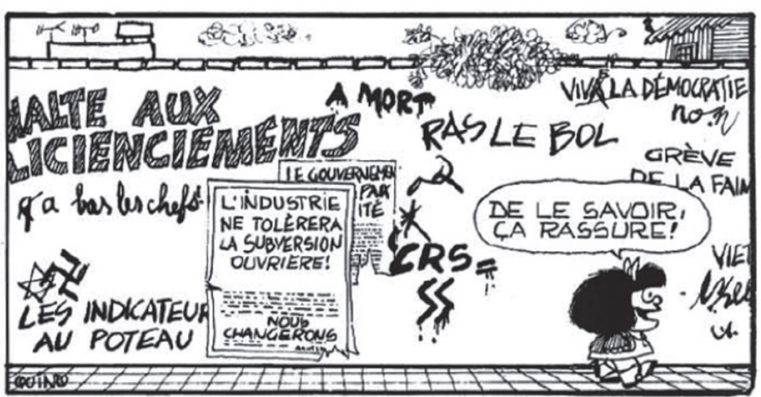

Figura 4. Ejemplo de referencias históricas. Fuente: Mafalda 1 por Joaquín Salvador Lavado y Mafalda intégrale, traducción por Jacques y Anne-Marie Meunier, viñeta número 122.

Uno de los desaciertos más notorios identificados en la traducción: "Venimos a hacerte un planteo, presidente" dicen Felipe y Manolito jugando. Lo cual se traduce como On vient te présenter un projet, président. En este sentido, se toma una acepción de la palabra planteo como una protesta en el texto original; sin embargo, en francés la palabra projet denota una propuesta o un plan, interrumpiendo con el sentido humorístico y semántico de la viñeta.

La mayoría de las referencias hace alusión al boom tecnológico, a la popularidad del idioma inglés y al crecimiento del capitalismo en general, tal es el caso donde se traducen las siglas de la empresa I.B.M. por la palabra ordinateur, computadora; "aprendamos inglés" y l’anglais à la maison; o "¿Quién puede sentir simpatía por plantas que se riegan con dinero?", que en francés pasa a Qui peut s'intéresser à ces campagnes qui s'arrosent avec de l'argent?

Dichos o proverbios. Esta categoría constituye tan solo un ejemplo: "Entre bueyes no hay cornadas", adaptada a Les loups ne se mangent pas entre eux. El primer dicho significa que entre personas allegadas no debería haber actitudes perjudiciales. Por otro lado, 
la definición del segundo dicho es "los canallas están siempre listos a ponerse de acuerdo cuando se trata de llegar a un mismo objetivo". En realidad, la diferencia es tan sutil que ni siquiera se podría afirmar que cambie el mensaje original.

Expresiones idiomáticas. Esta categoría constituye la más numerosa del análisis con más de 50 expresiones analizadas. Enseguida se describe una muestra representativa. Si bien se muestra una tira que mantiene una relación coherente entre imagen y texto, la traducción "iando con el ánimo por el suelo!" a j’ai le moral au tapis parece demasiado literal, la expresión más común a utilizar en francés es avoir le moral dans les chaussettes, con la cual se pudo haber respetado además la relación entre imagen y texto.

Mafalda advierte a su padre que se quedaría con la duda de lo que quería preguntarle por no estar dispuesto a escucharla. En su traducción, Mafalda usa una expresión idiomática en francés que literalmente sería algo así como "te dejaré sobre tu hambre". Por supuesto, es en el sentido figurado en el que esta frase toma sentido. Rester sur sa faim puede interpretarse como ver nuestras expectativas desilusionadas, así pues esta frase puede identificarse como una equivalencia.

En otro ejemplo, Mafalda incrédula pregunta a Felipe: “Ah, ¿sí?”. A lo cual los traductores sustituyeron por: mon œil ! Una expresión que significa que no creemos lo que se nos dice. La expresión "estamos fritos" más adelante, la cual parece ser muy similar en francés: on est cuit, ambas quieren decir estar sin esperanzas. Ras-le-bol y "estar harta" se considera un calco junto con "me revienta" por Ça commence à m'exaspérer, puesto que ambas expresiones tienen un significado similar. Debido a la cercanía lingüística de las lenguas es posible notar como muchas de estas expresiones son semejantes o provienen de situaciones culturales más o menos parecidas.

Con respecto al último caso por analizar en esta categoría, donde "la cigüeña" se vuelve una forma de eludir el tema o "maquillar" la realidad para que parezca más digerible. En su traducción, se utiliza la frase noyer le poisson, la cual quiere decir engañar a alguien con el fin de hacerle ceder u olvidarse del tema. Se respeta la intención del autor sin mayor inconveniente.

Nivel de dificultades de traducción de aspectos lingüísticos. La siguiente categoría acoge algunas de las dificultades que se podrían encontrar en la traducción de aspectos lingüísticos, tales como los juegos de palabras, las variantes dialectales y las onomatopeyas.

Juegos de palabras. Mafalda reclama a su madre que su leche tiene nata (Figura 5). "iSiempre igual! ¿Al servirla nunca te fijás si tiene nata?", cuestiona Mafalda con un semblante casi molesto. Después hay una viñeta sin texto en que se muestra a Mafalda mirando pensativa su tazón de leche. En el siguiente y último recuadro se muestra de nuevo a Mafalda con el mismo semblante del segundo recuadro y arremete de nuevo contra su madre: “¿O estás contra el control de la natalidad?". La elección de los traductores es Tu ne fais attention à la peau sur le lait!... Serais-tu contre la dépolitisation? El juego nata/natalidad y peau/dépolitisation se muestra claramente como un ejemplo de transcreación. 
En otro ejemplo, el papá de Mafalda pregunta entusiasmado "¿Hablan de plantas en ese programa? A lo que Mafalda responde "pero de plantas fabriles". Esto se traduce como On parle de campagne à la télévision? ... Des campagnes électorales. Plantas por plantas fabriles y campagne (campo/campaña) por campañas electorales.
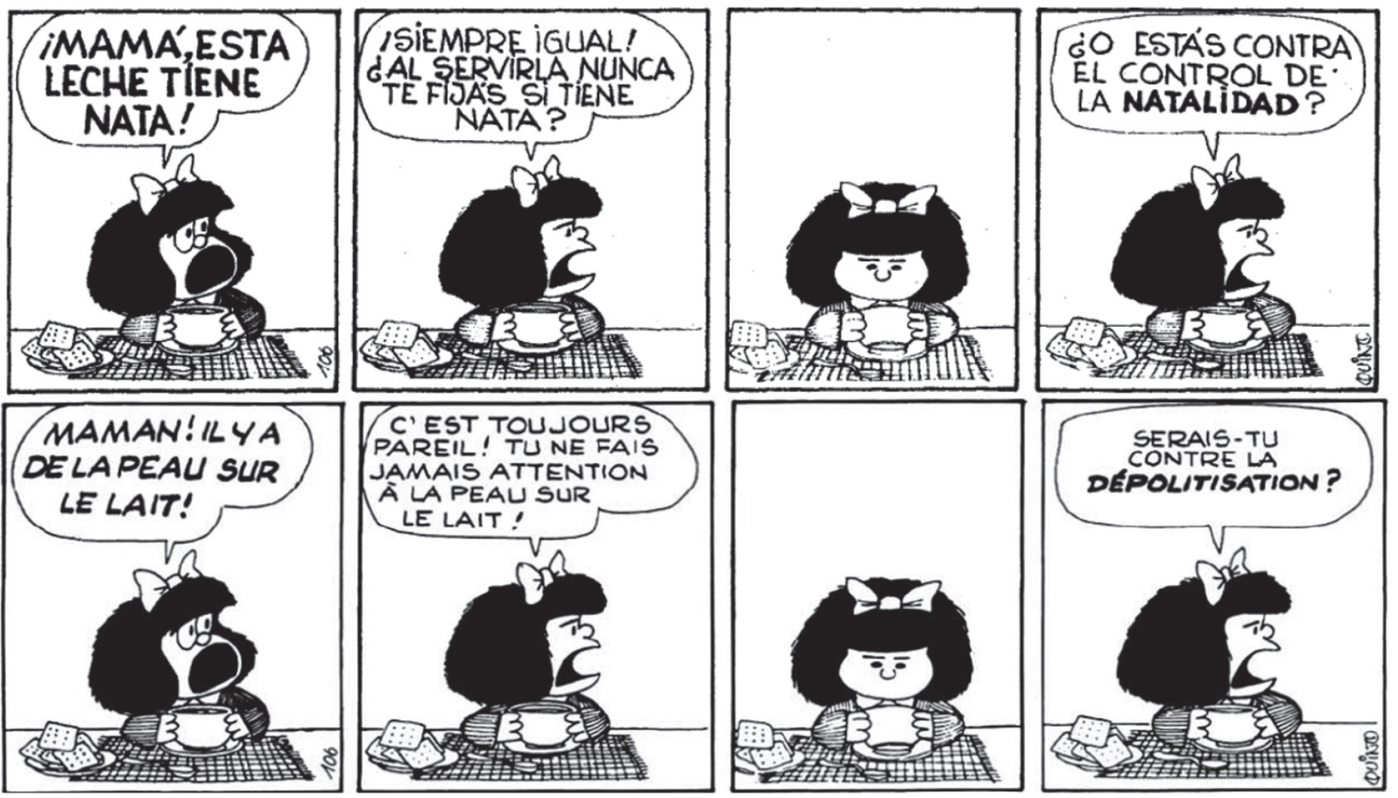

Figura 5. Ejemplo de juegos de palabras. Fuente: Mafalda 1 por Joaquín Salvador Lavado y Mafalda intégrale, traducción por Jacques y Anne-Marie Meunier, viñeta número 106.

Variantes dialectales. "iLa humanidad despachurrada!" La palabra "despachurrada" es, sin lugar a duda, de uso coloquial y puede llegar a tener varios significados. En este contexto se refiere a dejar a una persona confundida y sin saber qué decir o qué hacer. Ahora bien, Mafalda usa esta expresión para referirse al destino de la humanidad si una guerra atómica se desatara. En tal caso, la traducción utilizada por Jacques y Anne-Marie Meunier es: L'humanité en fumée!, lo cual se transfiere a desaparecer sin dejar rastro, evaporarse. Así, podríamos afirmar que la expresión, aunque cambia ligeramente en significado, transfiere el mensaje adecuadamente.
Dos casos en particular distinguen al español, más aún, al español en Latinoamérica. El uso del diminutivo es un recurso sobreexplotado y hasta complicado de categorizar dentro del idioma. Por un lado, el padre de Mafalda se acerca a ella en la noche al no poder dormir porque le ha dejado con la duda de que era aquello que quería preguntarle más temprano. Musita: “Mafaldita?". En este sentido, "apela a la voluntad del interlocutor para mover su voluntad a favor del hablante" (Giraldo, 1972, p. 72). Esta función es conocida como activo directo. Por otro lado, Mafalda se encuentra en la calle a una señora a la cual le cuestiona: 
“¿Usted es buena, señora?”. A lo que muy contenta responde: “YYo? ¡Sí, m’hijita, sí!". Aquí se podría decir que la función del diminutivo es un respeto afectuoso, una manera de referirse a las personas de manera casi condescendiente.

Un claro ejemplo de la influencia de la lengua y la cultura italiana en Argentina: “iMascalzone!" exclama Mafalda tras preguntarle a Felipe si era posible que utilizara la "defensa siciliana" en el juego de ajedrez. Esta palabra de claro origen italiano es una forma de decir "canalla" o "sinvergüenza" bien aceptada y comprendida por los argentinos. En su traducción, pierde la connotación cultural italiana al exclamar: Cocu! En su definición, se trata de un adjetivo para calificar a la persona que le ha sido infiel a su pareja, tomando un sentido humorístico diferente.

Finalmente, pregunta el papá de Mafalda a su esposa que sea ella la que le mencione a Mafalda que ya es hora de que reduzca la cantidad de tiempo que le dedica al televisor. A lo que la madre responde “¿Por qué no le decís vos?" En la traducción, se lee la respuesta: Dis-lui toi. La función interrogativa se transforma en el uso del imperativo. Es evidente que la cultura toma un gran peso en lo que refiere a esta viñeta. En los países de Latinoamérica existe una tendencia a diluir el discurso imperativo con el uso de la función interrogativa; por ejemplo, para pedirle a alguien que cierre la puerta se inclinarán más por decir “ipodrías cerrar la puerta?” antes que ordenar "cierra la puerta".

En general, los Meunier omiten la variante dialectal de Argentina en sus traducciones, traduciendo al francés estándar, tanto el léxico característico de este país como sus regionalismos y matices, que no son representados en Mafalda et ses amis.

Onomatopeyas. Por último, se muestran los resultados representativos de los sonidos escritos en las historietas analizadas. La tabla 2 presenta los resultados:

\section{Tabla 2}

\section{Muestra contrastiva de onomatopeyas}

\begin{tabular}{|c|c|c|}
\hline Joaquín Salvador Lavado & $\begin{array}{c}\text { Traducción Jacques y } \\
\text { Anne-Marie Meunier }\end{array}$ & Función \\
\hline ¡PTUÁJ! & POUAH! & Pide silencio \\
\hline ¡SSSHHH! & CHUT! & Grito \\
\hline ¡BUAAAA! ... & BOU-OUH! & Aclarándose la garganta \\
\hline ¡EJHÉM! & HUM! & Sonido del hipo \\
\hline ¡HÍC! & HIP! & . \\
\hline
\end{tabular}




\begin{tabular}{|c|c|c|}
\hline ¡JHÀ! & AH, AH ! & Risa burlona \\
\hline CHUIC & SMAC & Sonido de besos \\
\hline ¡SÑÍG! & SNIFF ! & Llanto \\
\hline ¡EPA!!! & HOLÀ! & Piden que se detenga \\
\hline ¡STUP! ¡STUP! & PFH ! PFH! & Escupiendo \\
\hline
\end{tabular}

Fuente: elaboración propia con extractos tomados de la historieta Mafalda 1 de Joaquín Lavado y la traducción al francés de Jacques y Anne-Marie Meunier.

\section{Análisis e interpretación de resultados}

Una vez analizados los resultados de las categorías presentes, es posible hacer ciertos enunciados. En cuanto a lo que refiere a los aspectos socioculturales, las canciones, por ejemplo, representan un gran reto para la labor del traductor; antes de proceder a traducir es necesario comprender la función o, en otras palabras, conocer cuál es el sema dominante que se pretende transmitir con dicha melodía. Habiendo aterrizado esta idea es posible concebir dentro de las fronteras de cada cultura una adaptación que logre recuperar el mensaje transmitido originalmente por el autor. Los Meunier logran transmitir el efecto humorístico y semántico en los dos casos citados debido a que buscaron melodías existentes en su propia cultura que produjeran un efecto similar en el lector.

Las costumbres están relacionadas con las formas de hacer comunidad y de relacionarse en sociedad. La cercanía cultural occidental permite en muchos casos realizar traducciones literales y en otros tomar decisiones lexicales puntuales para lograr la transferencia cultural, calcos o adaptaciones.
Las referencias literarias, políticas e históricas, por su parte, varían en el punto en que se transfiere o no el mensaje y en qué medida. En muchos casos, se hace omisión de algunas palabras debido a que no poseen un significado en la cultura meta o a que representan un obstáculo para comunicar el mensaje, perdiéndose así algunos elementos.

La Figura 4 es probablemente uno de los ejemplos de referencias más claros, ambas culturas toman esta oportunidad para presentar algunos de sus acontecimientos históricos más relevantes y que representan o representaron una lucha o una exigencia; se juega con la tipografía y con los mensajes en el muro de tal manera que se conserva el sentido de la viñeta.

En la categoría de expresiones idiomáticas existen múltiples conceptos abstractos o sumamente intrincados en la cultura a la que pertenecen. Los traductores echan a andar su creatividad buscando frases y expresiones que puedan mantener el sentido original, en muchas ocasiones les cuesta el sentido humorístico.

Los juegos de palabras son probablemente uno de los elementos lingüísticos más interesantes por interpretar dentro de las traducciones puesto que 
exigen, por parte del traductor, creatividad y flexibilidad, un conocimiento auténtico de la lengua y de la cultura tanto del texto original como del texto meta. Se logran transmitir efectivamente elementos fonéticos y culturales con gran astucia.

La mayoría de las variantes son traducidas en su versión estándar del francés. Por ejemplo, la palabra "opa" que en Argentina se utiliza para referirse a alguien bobo se lee como idiote, o el uso del diminutivo frecuentemente utilizado en América Latina para darle una connotación diversa a nuestras expresiones se omite en el texto meta; los traductores no transmiten la esencia detrás del personaje como portador de voz de su contexto, como sí se logra por ejemplo en las traducciones de Astérix al inglés: "la estrategia que siguen los traductores ingleses es hacer que los personajes bretones hablen inglés con características dialectales escocesas o galesas" (Muñoz y Buesa, 2010).

Por último, las onomatopeyas, los resultados demuestran que la trasferencia de los sonidos y las grafías se mantiene en ambos textos, cumpliendo así con su función.

\section{Discusión}

A partir de los resultados previamente descritos y de su interpretación es posible dar forma a una serie de planteamientos sobre el análisis expuesto. El objetivo que este trabajo de investigación se planteaba era, inequívocamente, afanoso: conocer todos y cada uno de los elementos culturales contenidos en una historieta con más de cinco décadas de antigüedad y cómo estos elementos fueron traducidos en su versión homóloga al idioma francés pasadas más de dos décadas entre ellas.

Con la hipótesis resultante de la principal pregunta de esta investigación se afirmaba que las traducciones realizadas por Jacques y Anne-Marie Meunier en francés de las tiras cómicas de Quino perdían, en cierta medida, algunos elementos culturales en el proceso de traducción. Esto se comprueba al compararlas con las originales, identificando los elementos lingüísticos y culturales trastocados.

En cierta medida, después de este análisis contrastivo, se podría argumentar que esta hipótesis se ha cumplido; no obstante, es necesario delimitar algunas aclaraciones pertinentes. Debido a la cercanía en las familias lingüísticas de ambas lenguas fue evidente percibir una cierta comodidad por parte de los traductores al realizar en su mayoría traducciones literales o nociones equivalentes. La transcreación y la adaptación fueron en algunos casos la excepción a esta noción.

En cuanto al análisis de las dificultades de los aspectos socioculturales, se demostró, a partir de los resultados arrojados, una increíble gama de elementos culturales a los que los traductores tuvieron que recurrir para superar la distancia cultural entre el texto original y el texto meta. El uso de sustituciones culturales para acercar la historieta al público francoparlante con referencias gastronómicas, históricas, literarias y hasta cinematográficas que verdaderamente fueran auténticas de la propia sociedad a la que se dirigían los traductores.

La segunda parte, el análisis de las dificultades de los aspectos lingüísticos, representó de igual manera un 
reto debido a las diversas herramientas utilizadas por los traductores, tales como el dominio de los semas y los fonemas en ambos idiomas para hacer frente a los juegos de palabras y las onomatopeyas.

Con todo, si bien existen elementos culturales que se perdieron en el camino de la traducción debido a su inexistencia o poca relevancia en la cultura meta, por ejemplo, el dialecto argentino con su léxico característico y registros, otros nuevos elementos resurgieron y tomaron su lugar para crear nuevos significados y paradigmas, en muchos casos igualmente ingeniosos a los contenidos originales.

Por último, se hace una invitación a todos los traductores a considerar una nueva concepción de lo que se requiere para realizar una traducción cultural que pueda ser figurada como acertada y vigente. Dicha concepción es incluyente de una traducción que no transmita estrictamente todos los elementos culturales pero que, aun así, logra un contenido auténtico y significativo para la cultura meta.

\section{Bibliografía}

Claramonte, M. C. (2009). A vueltas con la traducción del siglo XXI. MonTI. Monografías de Traducción e Interpretación, 1, pp. 49-58.
Libedinsky, J. (2001, 16 de marzo). Mafalda cumplió años y será traducida al inglés. La Nación.

AltaLingua. (2018, 4 de mayo). Mafalda en todos los idiomas. Obtenido de AltaLingua Traducciones. En https://altalingua.wordpress.com/2016/01/26/ mafalda-en-todos-los-idiomas/

Buzelin, H. (2004). Henry, J. (2003). La traduction des jeux de mots. Presses de la Sorbonne Nouvelle, pp. 387-391.

Dell'Isola, S. (2014, 19 de septiembre). Mafalda cumple 50. Las siete claves que la definen y la hicieron emblemática. La Nación, p. 14.

Giraldo, J. J. (1972). Funciones del diminutivo en español: ensayo de clasificación. Centro Virtual Cervantes, pp. 71-88.

Muñoz, M. y Buesa, C. (2010). Ils sont fous ces traducteurs: La traducción del humor en cómic de Astérix. Universidad de Zaragoza, pp. 419-476.

Pym, A. (2016). Teorías contemporáneas de la traducción. Tarragona, España: Intercultural Studies Group. 